the present study found neither greater resistance to extinction following discrimination training ( $E-2$ vs $N-D)$ nor positive induction ( $\mathrm{E}-2$ vs $\mathrm{E}-\mathrm{I}$ ).

The different results in the two replications suggest a third variable that might underlic the mixed results in the literature. The obtained difference must be a function of the baseline training used in the first replication. The effect may be due to switching from baseline equal reinforcement to discrimination differential reinforcement or from the 20 extra sessions of reinforcement. In comparing the two replications the discrimination groups are very similar in extinction. It is the difference between the two nondiscrimination groups that suggests the duration-of-training variable. The N-D group extinguished more rapidly in the second replication. It would appear that 22 sessions of simple acquisition led to faster extinction than 42 sessions. Different amounts of discrimination training (see D’Amato, Schiff, \& Jagoda, 1962) were not evaluated in the present study, but also seems to be an important variable.

The present analysis suggests that there is not a simple relationship between discrimination training and resistance to extinction. At least, the following variables and their interactions have to be considered: (1) the schedule of reinforcement used in establishing response rate: (2) the method of testing post-discrimination extinction; and (3) the duration of training.
REIERFNCYS

BIRCH. D. ALLISON, J. K.. \& HOCSI: R. T lixtinction performance following diserimination training. Journal of lixperimental Psychology. 1963,65.148.155.

D'AMATO, J. A., SCHIl F. D. \& JAGODA. H. Resistance to extinction after varying amounts of discrimination or nondiscrimination instrumental training. Journal of Experimental Psychology, 1962, 64, 526-532.

JENKINS, H. M. The effects of discrimination training on extinction. Journal of Fxperimental Psychology, 1961, 61, 111-121. MoCROSKERY, J. H. Extinguishing, abolishing, and reversing discriminative responding. inpublished doctoral dissertation, University of Kentucky, 1969.

REYNOLDS, G. S. Induction, contrast, and resistance to extinction. Journal of the Experimental Analysis of Behavior, 1968, 11 , $453-457$

SENF, G. M., \& MILLER, N. E. Evidence for positive induction in disrimination learning. Journal of Comparative \& Phsyiological Psychology, 1967, 64, 121-127.

SKINNER, B. 1 . The behavior of organisms. New York: Appleton-Century-Crofts, 1938.

\section{NOTES}

1. Now located at State Liniversity College, Oswego. N.Y. 13126.

2. The numeral ' 1 ' as in $S 1$ refers to rointorement. and the mumeral ' 2 ' as in $S 2$ refers to nonrsinforcment. The let ter " $\mathrm{S}$ " refer to the stimulus. and the numerals refer to reinforcement contingencies associated with that stimulus during the two main phases of the experiment. For example, S12 means that this stimulus is presently associated with nonreinforcement and that in the preceding phase this same stimulus was associated with reinforcement.

\title{
Motivational effects of differential magnitudes of reinforcement
}

JEFF S. TOPPIVG, JOHV M. COLE, MARCLI, H. MATAMOROS, and CHLCK H. LINENBERGER, University of Portland, Portland, Ore. 97203

The present study attempted to determine if a relatively small reinforcement magnitude, when contrasted with a large magnitude, would produce a motivational effect similar to that observed when nonreinforcement is contrasted with reinforcement. Results indicated that contrasting magnitudes produce more activity following the relatively smaller of two reinforcement magnitudes.

The hypothesis that nonreinforcement following reprated reinforcement has active motivat onal properties has received much experi nental support. Goodrich (1959) and Spence (1960) have employed this notion to account for increased acquisition sffects under partial reinforcement (PK), while Gallup (1965) and Azrin, Hutchinson, \& Hake (1966) have obtained results that suggest that aggressive responding can be evoked by nonreinforcement during both acquisition and extinction. Further support comes from studies on the frustration effect (McCain \& McVean. 1967; Wagner, 1959) in which increased running speeds are found in the second alley of a double runway following nonreinforced, as opposed to reinforced, trials in the goalbox of the first alley.

Using a somewhat different approach to this problem, Gallup \& Altomari (1969) have provided additional positive evidence. They trained rats to traverse a straight alley under conditions of PR. Upon termination of an alley trial, Ss were removed and placed in an open field, where such measures of activity as ambulation, rears, and climbs were obtained. Results showed that Ss displayed more activity following nonreinforced trials than following reinforced trials. It was concluded that nonreinforcement may produce a more potent and durable motivational state than previously imagined. Since the motivation was measured in a physically separate open field, these results suggest that nonreinforcement may produce a general motivational state that is relatively independent of stimuli associated with the primary reinforcement. Gallup \& Hare (1969) replicated these findings and also discovered that (1) there was more activity in extinction for a continuous reinforcement (CR) group than for a $P R$ group, and (2) more activity occurred after reinforced trials in a PR group than after comparable trials in a CR group. This latter finding prompted the interpretation that the motivational effects of nonreinforcement become conditioned to the goalbox cues so that exposure to these cues on reinforced trials also results in enhanced motivation. This result is also in accord with the notion of behavioral contrast (Reynolds, 1961).

In the previously mentioned experiments, some magnitude of reinforcement was always contrasted with nonreinforcement, with the aim being to demonstrate the motivational properties of the latter. In the present experiment, differential magnitudes of reinforcement were contrasted using the Gallup \& Altomari (1969) procedure, in order to determine whether or not the relatively smaller magnitude would produce a motivational effect similar to that observed with nonreinforcement.

\section{METHOD}

The Ss were 12 Mongolian gerbils, six males and six females, ranging in age from 140 to 175 days at the start of the experiment.

The apparatus consisted of a gray straight alley and a physically separate circular open field. The straight alley measured $76 \times 4 \times 9$ in., with $141 / 2$-in. start, $47 \frac{1}{2}$-in. run, and 14-in. goal sections, separated by wooden guillotine doors. A 2 -in. glass caster wrapped with black tape was employed as the food cup and was located on the floor against the end wall of 
the goal section. Quarter-inch wire mesh covered the top of the alley. The white open field had a 3 -ft diameter and 1-ft-high sides, and the floor was divided in to 4 -in. squares by thin black lines.

Two weeks prior to training, Ss were placed on a 22-h food-deprivation schedule with free access to water, and this schedule was maintained throughout the experiment. This procedure maintained Ss at approximately $85 \%$ of their ad lib weights. Beginning 1 week before training, Ss were given daily 5 -min exploration periods in the unbaited alley in order to habituate them to the movable guillotine doors. Following daily alley habituation, Ss received daily 3-min sessions in the open field, and the number of squares crossed was counted and recorded for each $S$. Each groups of two males and two females each.

During the 14 days of acquisition training, Ss received six massed trials daily, all of which were reinforced. Reinforcement consisted of 37-mg Noyes pellets, with large (L), medium (M), and small (S) magnitudes being five, three, and one pellet, respectively. The individual groups received only two of these magnitudes, thereby producing groups hereafter designated as LS, LM, and MS. For each group, the two designated reinforcement magnitudes occurred on $50 \%$ of the daily trials, and the sequence was randomized daily, with the exception that the relatively larger magnitude always occurred on the final daily trial. All groups received the same daily sequence of the relatively smaller ( - ) and relatively larger (t) magnitudes, and the order of running Ss was randomized and constant across days.

On each trial, $S$ was placed in the start section, the guillotine door separating the start and run sections was raised, and $S$ was allowed to run to the food cup. After $S$ entered the goal section, the guillotine $S$ 's activity scores for the last 2 days were used to assign the $S s$ into three matched

door separating the goal and run sections was lowered to prevent retracing. As soon as the reinforcement had been completely consumed and $S$ had stopped chewing, $S$ was removed from the goal section and placed at the center of the open field. Ss were given $30 \mathrm{sec}$ in the open field, during which time the number of squares crossed was counted. The $S$ was then returned to the start section of the alley for another trial. While $\mathrm{S}$ waited in the start section, open field activity data was recorded for the preceding trial, the food cup was re-baited, and both pieces of apparatus were cleaned with a damp sponge to remove any urination, defecation, or other distracting stimuli. This procedure resulted in an intertrial interval of approximately 1 min. Following the final daily trial, $S$ was returned to his home cage and given $2 \mathrm{~h}$ of free access to food.

\section{RESULTS AND DISCUSSION}

The mean number of squares crossed for each $S$ during both - and + trials was computed, and the total of these mean scores, for each group during the entire course of training, is presented in Table 1.

A 3 (groups) by 2 (reinforcement magnitudes) by 5 (days) repeated-measures ANOVA was performed on the mean activity scores of Days 10-14. The main effect of reinforcement magnitude ( $-v s+$ ) was found to be highly significant $(F=59.96, d f=1 / 9, p<.001)$, indicating that Ss were more active after trials with the relatively smaller reinforcement magnitude. The main effect of days was also significant $(F=9.53, \mathrm{df}=4 / 36$, $\mathrm{p}<.01$ ), indicating that, in general, Ss became more active as training progressed. Finally, a significant Reinforcement Magnitude by Days interaction $(F=3.45$, $\mathrm{df}=4 / 36, p<.05)$ indicates that the difference in activity following - and + trials varied over days. A Newman-Keuls test showed that this significant interaction was due to the fact that all of the five daily mean differences ( $-v s+)$ were

Table I

Open Field Activity Scores

\begin{tabular}{rrrrrrr}
\hline & \multicolumn{2}{c}{ Group LS } & \multicolumn{2}{c}{ Group LM } & & Group MS \\
\hline Days & + & - & + & - & + \\
1 & 60.00 & 60.00 & 56.33 & 59.00 & 62.67 & 53.00 \\
2 & 46.67 & 58.33 & 55.67 & 38.33 & 53.00 & 53.67 \\
3 & 49.67 & 51.00 & 44.33 & 54.00 & 45.33 & 50.33 \\
4 & 62.67 & 61.00 & 59.33 & 59.33 & 52.33 & 67.67 \\
5 & 52.67 & 61.00 & 50.33 & 57.67 & 73.00 & 71.33 \\
6 & 60.00 & 66.33 & 49.67 & 59.00 & 69.00 & 88.00 \\
7 & 57.00 & 57.00 & 43.00 & 49.67 & 57.67 & 79.33 \\
8 & 48.67 & 48.33 & 62.33 & 57.00 & 65.67 & 66.00 \\
9 & 52.00 & 76.67 & 54.67 & 71.01 & 70.66 & 76.00 \\
10 & 38.33 & 57.33 & 32.34 & 43.34 & 44.00 & 53.32 \\
11 & 48.33 & 61.67 & 40.99 & 52.00 & 50.99 & 67.00 \\
12 & 55.33 & 72.66 & 49.00 & 58.00 & 57.66 & 67.33 \\
13 & 58.66 & 69.01 & 44.67 & 45.66 & 61.67 & 72.67 \\
14 & 66.34 & 96.34 & 73.00 & 95.33 & 67.66 & 87.33 \\
\hline
\end{tabular}

significantly different $(p<.05)$ from each other, with the exception of Days 10 and 11. No other effects were statistically significant.

The results obtained in the present study extend previous findings of Gallup \& Altomari (1969) and Gallup \& Hare (1969) by showing that a relatively smaller reinforcement magnitude, when contrasted with a larger magnitude, can produce a motivational effect similar to that observed when nonreinforcement is contrasted with reinforcement. Although the experimental situations are markedly different, the present results would not be predicted by McHose (1969), who concluded that there was a definite functional discrepancy between nonreinforcement and relatively small reinforcement.

In addition, it was predicted that a significantly greater motivational effect would occur in Group LS than in Groups LM and MS. While the differences (see Table 1) were in the expected direction, the Groups by Reinforcement Magnitude and Groups by Reinforcement Magnitude by Days interactions were not significant, indicating the lack of any significant motivational differences between groups. This nonsignificant result right be partially attributed to the small difference between groups in the number of pellets employed for the relatively smaller and larger reinforcement magnitudes.

\section{REFERENCES}

AZRIN, N. H., HUTCHINSON, R. R., \& HAKE D. F. Extinction-induced aggression. Joumal of the Experimental Analysis of Behavior, $1966,9,191-204$

GALLUP, G. G. Aggression in rats as a function of frustrative nonreward in a straight alley. Psychonomic Science, 1965, 3, 99-100.

GALLUP, G. G., \& ALTOMARI, T. S. Activity as a postsituation measure of frustrative nonreward. Journal of Comparative \& Physiological Psychology, 1969, 68, 382-384.

GALlUP, G. G., \& HARE, G. K. Activity following partially reinforced trials: Fvidence for a residual frustration effect duc to conditioned frustration. Psychonomic Science, $1969,16,41-42$.

GOODRICH, K. P. Performance in different segments of an instrumental response chain as a function of reinforcement schedule. Journal of Experimental Psychology, 1959, 57, 57-63.

McCAIN, G., \& McVEAN, G. Effects of prior reinforcement or nonreinforcement on later performance in a double alley. Journal of Experimental Psychology, 1967, 73, 620-627.

McHOSE, J. H. Role of frustration in the development of relative and absolute $S$ discrimination contrast effects. Journal of Experimental Psychology, 1969, 81, 256-260.

REYNOLDS, G. S. Behavioral contrast. Journal of the Experimental Analysis of Behavior, $1961,4,57-71$.

SPENCE, K. W. Behavior theory and learning. Englewood Cliffs, N.J: Prentice-Hall, 1960.

WAGNER, A. R. The role of reinforcement and nonreinforcement in an "apparent frustration effect." Joumal of Experimental Psychology, $1959,57,130-136$. 